\title{
Fibrinogen Effects of Erythrocyte Nitric Oxide Metabolism in Presence of Timolol
}

\author{
Carlota Saldanha*, Teresa Freitas and Ana Santos Silva-Herdade \\ Institute of Biochemistry, Institute of Molecular Medicine, Faculty of Medicine University of Lisbon, Av. Prof. Egas Moniz, 1649-028 Lisbon, Portugal
}

\begin{abstract}
Timolol inhibits erythrocyte membrane acetylcholinesterase (AChE) enzyme activity originates less active enzyme complex forms unable to change the normal values of the nitric oxide (NO) efflux from erythrocytes. The active complex resulting from acetylcholine (ACh) binding to AChE induces higher values of NO efflux. Increased values of $\mathrm{NO}$ efflux were also observed in presence of high fibrinogen (Fib) concentration with return to normal levels of $\mathrm{NO}$ efflux when $\mathrm{ACh}$ is added. Association between NO efflux and erythrocyte deformability values has been evidenced.
\end{abstract}

The objective of this study was to evaluate the effects of high fibrinogen concentration on the erythrocyte NO metabolism and deformability in presence of timolol without and plus adenylyl cyclase or plus guanylyl cyclase inhibitors.

Blood samples from healthy donors were divided in aliquots without (control) or with Fib or Fib plus timolol in absence or presence of adenylyl cyclase inhibitor (MDL) or guanylyl cyclase inhibitor (Ly). Erythrocyte deformability (ED), NO efflux, S-nitrosoglutathione (GSNO), nitrite and nitrate values were evaluated by referenced methods

Fib increased the values of NO efflux under the influence of timolol or MDL. Increased values of NO efflux, GSNO, nitrites and nitrates where obtained in presence of Fib plus timolol plus MDL in relation to control to Fib or Fib plus MDL aliquots. Increased nitrites and nitrates values where obtained in presence of Fib plus timolol plus Ly in relation to control, to Fib and Fib plus Ly. Only at high shear stress increased ED values was observed in Fib plus timolol in absence and presence of MDL.

In conclusion fibrinogen plus timolol increased ED at high shear stress without or with MDL. Fibrinogen increased NO efflux from erythrocytes and GSNO, nitrite and nitrate levels in presence of timolol plus guanylyl cyclase inhibitor.

\section{Introduction}

The plasma fibrinogen (Fib) molecule participates in fibrin formation, red blood cells (RBCs) aggregation and when at high concentrations is known as an inflammatory acute phase protein [1-3]. Fibrinogen binding to erythrocyte CD47 receptor protein belonging to $\mathrm{Rh}$ complex that stablish contact with 4.2 cytoskeletal protein which interact with band 3 protein $[4,15]$. Mimicking, in vitro hyperfibrinogenemia increased values of nitric oxide (NO) efflux from RBCs dependent on band 3 protein phosphorylation degree and lower cyclic adenosine monophosphate (cAMP) concentration was evidenced [6,7]. Maintaining high Fib concentrations, the levels of NO efflux from $\mathrm{RBCs}$ normalize by the presence of acetylcholine (ACh) binding to RBCs membrane acetylcholinesterase (AChE) [8]. The explanation comes from the signal transduction pathway initiate by the AChE-ACh active complex forms $[9,10]$. So, activation of protein kinase C (PKC) inhibits by phosphorylation the phosphodiesterase (PDE3) resulting increased amount of cAMP concentration that abolish the higher NO efflux induced by hyperfibrinogenemia observed in absence of ACh [8-10]. While ACh increase erythrocyte deformability (ED) at high shear stress, high Fib concentrations increase it at low shear stress under band 3 dephosphorylated by protein tyrosine kinase (PTK syk) inhibition [11,12].

Timolol is a moderate inhibitor of erythrocyte $\mathrm{AChE}$ and normal values of NO efflux from RBCs has been verified under AChE-timolol complex, which are lower than those evaluated when AChE-ACh active complex forms are presented in eryrthrocytes [13-16]. Increased NO efflux, S-nitrosoglutathione (GSNO), nitrite and nitrate values were evidenced in presence of timolol plus adenylyl cyclase inhibitor (MDL) [17]. It was observed an increase in ED at high shear stress induced by the simultaneous presence of timolol and band 3 protein dephosphorylation by PTK syk inhibitor [17].

The aim of this study was to evaluate the effects of high fibrinogen concentration on the erythrocyte NO metabolism and deformability in presence of timolol without and plus adenylyl cyclase or plus guanylyl cyclase inhibitors.

\section{Material and Methods}

\section{Chemicals}

Acetycholine chloride,adenylyl cyclase inhibitor (MDL hydrochloride ), guanylyl cyclase inhibitor (Ly-83583,) were purchase

Correspondence to: Carlota Saldanha, Institute of Biochemistry, Institute of Molecular Medicine, Faculty of Medicine University of Lisbon, Av. Prof. Egas Moniz, 1649-028 Lisbon, Portugal, E-mail: anarmsilva@medicina.ulisboa.pt

Key words: Fibrinogen, erythrocyte, timolol, nitric oxide; adenylyl cyclase; guanylyl cyclase

Received: March 12, 2018; Accepted: March 19, 2018; Published: March 29, 2018 
from Sigma (St Louis, MO, USA). The human fibrinogen was purchased from Sigma (Poole, UK). The chosen concentrations for fibrinogen were based on its physiological levels and on previous studies [18]. Timolol maleate was purchased from Laboratórios Químico- Farmacêuticos, Chibret, Lda. Nitrate reductase from Aspergillus Niger, NADPH (tetra sodium salt), sodium nitrate, sodium nitrite and atropine were all from Sigma Chemical Co., St Louis, MO, USA. The Griess Reagent kit was purchased from Molecular Probes, Eugene, USA. Sodium chloride was purchased from AnalaR (UK) and chloroform and ethanol 95\% from MERCK, Darmstadt, Germany. Blood samples were collected into tubes BD Vacutainer TM with Lithium heparin $(17 \mathrm{UI} / \mathrm{mL})$ as an anticoagulant.

\section{Blood sampling and experimental model}

This in vitro study was performed under the protocol established with the Portuguese Institute of Blood in Lisbon. All males donors $(\mathrm{N}=10$; aged between 30 and 40 years old) were duly informed and signed their agreement.

Blood samples were collected into tubes with lithium heparin (17 $\mathrm{IU} / \mathrm{ml}$ ) as anticoagulant. Total blood was divided into eight aliquots of $1 \mathrm{ml}$ each and centrifuged at $11000 \mathrm{rpm}$ (Biofuge 15 centrifuge, Heraeus) during 1 minute at room temperature. Then $10 \mu \mathrm{L}$ of plasma were replaced by the same volume of either physiological serum (control aliquot) or fibrinogen $\mathrm{NaCL}$ isotonic solutions $\mathrm{pH}$ 7,4 (30 $\mathrm{mg} / \mathrm{dL}$ ) or timolol or Fib plus MDL or Fib plus Ly or Fib plus timolol or Fib plus MDL plus timolol or Fib plus Ly plus timolol plus each inhibitor, so that the final concentration of timolol and each inhibitor in the whole blood aliquots was $10-5 \mathrm{M}$. Besides other concentrations $(5 \times 10-5,10-6$ and $5 \times 10-6 \mathrm{M})$ were tested, no significant alterations were observed with these concentrations in relation to $10-5 \mathrm{M}$. Then the blood sample was homogenized by gently inversion incubated for 15 minutes at room temperature. At the end of incubation samples were centrifuged and plasma removed for fibrinogen concentration assessment. Then erythrocyte deformability, NO efflux from RBC and the other NO derivative molecules were determined following the methods described next.

\section{Determination of fibrinogen concentration by clot-based technology}

Plasma fibrinogen concentrations were evaluated using the Fibritimer BFT*//Analyser (Dade Behring, Marburg GmbH, Germany) based on the Clot technology.

\section{Measurement of erythrocyte NO efflux, nitrite, nitrate, GSNO and peroxynitrite}

Following incubation, blood samples were centrifuged and sodium chloride $0.9 \%$ with a $\mathrm{pH} 7.0$ was added to establish a hematocrit of $0.05 \%$. The suspension was mixed by gentle inversion.

In order to quantify amperometric NO quantification, we used the amino-IV sensor (Innovative Instruments Inc. FL, USA), according to the method described previously [19] NO diffuses through the gas-permeable membrane tripleCOAT of the sensor probe and it is then oxidized at the working platinum electrode, resulting on an electric current. The redox current is proportional to the NO concentration outside the membrane and was continuously monitored with a computerized inNOTM system, (with a software version 1.9, Innovative Instruments Inc., Tampa, FL, USA). Calibration of the NO sensor was performed daily. For each test, the NO sensor was immersed vertically in the erythrocyte suspension vials and allowed to stabilize for $30 \mathrm{~min}$ to achieve basal NO levels. $30 \mu \mathrm{l}$ of acetylcholine was added to the erythrocyte suspension samples in order to achieve final concentrations of $10 \mu \mathrm{M}$ of ACh and NO. Data was recorded from the constantly stirred suspensions at room temperature.

The measurement of nitrite/nitrate concentration was performed using the spectrophotometric Griess method as already described [20], after submitting the pellet of each centrifuged blood sample to haemolysis and haemoglobin precipitation. Haemolysis was induced with distilled water and hemoglobin precipitation with a mixture of ethanol and chloroform $(5 \mathrm{v} / 3 \mathrm{v})$. The nitrite concentrations were measured with the spectrophotometric Griess reaction, at $548 \mathrm{~nm}$. For nitrate measurement, this compound was first reduced to nitrites in presence of nitrate reductase [21].

S-nitrosoglutathione (GSNO) was measured utilizing colorimetric solutions containing a mixture of sulfanilic acid ( $\mathrm{B}$ component of Griess reagent) and NEDD (A component of Griess reagent), consisting of $57.7 \mathrm{mM}$ of sulfanilic acid and $1 \mathrm{mg} / \mathrm{mL}$ of NEDD were dissolved in phosphate-buffered solution (PBS; pH 7.4). To constitute the $10 \mathrm{mM} \mathrm{HgCl} 2$ (Aldrich) mercury ion stock solutions were prepared in $0.136 \mathrm{~g} / 50 \mathrm{~mL}$ of dimethyl sulfoxide (DMSO) (Aldrich). GSNO was diluted to the following desired concentrations: 7,5 $\mu \mathrm{M} ; 15 \mu \mathrm{M} ; 30 \mu \mathrm{M}$; $45 \mu \mathrm{M} ; 60 \mu \mathrm{M} ; 120 \mu \mathrm{M} ; 240 \mu \mathrm{M} ; 300 \mu \mathrm{M}$ in the colorimetric analysis solutions. Various concentrations of mercury were then added to a final concentration of $100 \mu \mathrm{M}$. Following gentle shaking, the solution was let to stand for twenty minutes. A control spectrum was measured by spectrophotometry at $496 \mathrm{~nm}$ against a solution without mercury ion. $300 \mu \mathrm{L}$ of erythrocyte suspensions were added to the reaction mixture and GSNO concentrations were obtained as already described [22].

\section{Erythrocyte deformability}

The erythrocyte deformability (ED) for different shear stress $(0.30$, $0.60,1.20,3.00,12.00,30.00$ and $60.00 \mathrm{~Pa}$ ) was determined by using the Rheodyn SSD shear stress diffractometer from Myrenne GMBH (Roentgen, Germany), and erythrocyte deformability is expressed as the elongation index (EI) in percentage (20). The Rheodyn SSD diffractometer determines RBC deformability by simulating the shear forces exerted by the blood flow and vascular walls on the erythrocytes. Erythrocytes are suspended in a viscous medium and placed between a rotating optical disk and a stationary disk. A well-defined shear force is exerted upon the suspension which forces the erythrocytes to deform to ellipsoids and align with the fluid shear stresses. If a laser beam is allowed to pass through the erythrocyte suspension a diffraction pattern appears on the opposite end. That diffraction pattern will be circular with resting erythrocytes, but becomes elliptical when the erythrocytes are deformed by shear. The light intensity of the diffraction pattern are measured at two different points (A and B), equidistant from the center of the image. The erythrocyte elongation index (EEI) is expressed as a percentage, obtained according the following formula: $\mathrm{EEI}(\%)=[(\mathrm{A}-$ $\mathrm{B}) /(\mathrm{A}+\mathrm{B})] \times 100[23]$.

This study was performed in accordance with the ethical guidelines of Clinical Hemorheology and Microcirculation [24].

\section{Statistical analysis}

Data are expressed as mean values \pm SD. Student's paired t-tests were used to compare values between different samples of erythrocyte suspensions. Statistical analysis was conducted using the Statistical Package from the Social Sciences (SPSS; version 16.0). One-way analysis of variance and paired t-tests were applied to assess statistical significance between samples. Bonferroni post-hoc tests were 
conducted when appropriate. Statistical significance was set at a $\mathrm{p}<0.05$ level.

\section{Results}

Using blood samples instead of erythrocytes suspension we mimics the experimental model of hyperfibrinogenemia and $510 \pm 10 \mathrm{mgdL}$ was the Fib concentration in the aliquots.

Effects of fibrinogen on erythrocyte deformability under the absence an presence of timolol and timolol plus adenylyl and guanylyl cyclase inhibitors

No significantly values of erythrocyte deformability at all shear stress were observed in all blood aliquots in relation to the control one at exception of two of them respectively Fib plus timolol in absence $(49.2 \pm 3.99$ vs $47.7 \pm 3.11)$ and presence of MDL $(48.9 \pm 3.46$ vs 47.7 $\pm 3.11)$ Table 1 .

Effects of fibrinogen on nitric oxide metabolism e under the absence an presence of timolol and timolol plus adenylyl and guanylyl cyclase inhibitors

Table 2 shows that the values of NO efflux from erythrocytes, obtained in presence of MDL, or timolol or Fib plus Ly do not show significant changes in relation to the control blood aliquots. Significantly increased values $(\mathrm{p}<0.02)$ in relation to control aliquots $(1.09 \pm 0.11)$ were obtained when Fib is present with MDL (1.50 \pm 0.42$)$ or with timolol $(1.23 \pm 0.14)$ or with MDL plus timolol $(1.49 \pm 0.36)$ or with Ly plus timolol (1.49 \pm 0.36$)$. Comparing the NO efflux values in presence of Fib plus MDL plus timolol (1.49 \pm 0.36$)$ in relation to the Fib aliquot $(1.23 \pm 0.11)$ a significantly $(\mathrm{p}<001)$ difference was obtained Table 2

The values of GSNO inside the erythrocytes increase significantly $(p<0.02)$ in all treated blood aliquots in relation to the control aliquots
Table2. It is highlight, that the value of GSNO of Fib plus MDL plus timolol $(12.28 \pm 0.70)$ present significantly $(\mathrm{p}<0.03)$ increased in relation to Fib $(9.13 \pm 0.34)$ and to Fib plus MDL blood aliquot $(10.86 \pm 1.29)$ $\mathrm{p}<0.05$ Table 2 .

Regarding nitrate values in all treated blood aliquots in relation to the control ones they are all significantly $(\mathrm{p}<0.01)$ increased Table 2. When Fib is added to MDL plus timolol the values of nitrate $(12.28 \pm 0.70)$ were increased significantly $(\mathrm{p}<0.001)$ and $(\mathrm{p}<0.03)$ also in relation to Fib $(10.15 \pm 0.64)$ and to Fib plus MDL $(11.50 \pm 0.84)$ respectively.

The variation of the nitrites values obtained in treated blood aliquots in relation to the control aliquots is almost similar to the nitrate with exception of absence changes in the blood aliquots values obtained in presence of Fib or MDL Table 2. When Fib is added to MDL plus timolol the values of nitrite $(12.30 \pm 0.59)$ were increased significantly $(\mathrm{p}<0.001)$ and $(\mathrm{p}<0.03)$ also in relation to Fib $(9.45 \pm 0.34)$ and to Fib plus MDL $(11.10 \pm 0.82)$ respectively.

\section{Discussion}

The present study confirms that erythrocyte deformability is not changed by high Fib concentration. Our previously data showed increase of $\mathrm{ED}$ values when high $\mathrm{Fib}$ is under band 3 protein dephosphorylated state or in presence of AChE- ACh active complex form $[8,12]$. The ED values are not affected by high Fib levels in absence or presence of timolol plus guanylyl cyclase inhibitor Ly in any shear stress Table 1. In absence of fibrinogen the AChE-timolol less active complex plus MDL blood aliquots did not influence ED values [17].

Erythrocyte deformability increased in blood aliquots with Fib plus timolol in absence or presence of adenylyl cyclase inhibitor

Table 1. Values (Mean \pm SD) of erythrocyte elongation index at different shear stress obtained in absence (Control) and presence of fibrinogen (Fib) or Fib plus timolol or Fib plus adenylyl cyclase inhibitor (MDL), or plus guanylyl cyclase inhibitor(Ly).

\begin{tabular}{|c|c|c|c|c|c|c|c|c|c|}
\hline \multirow[b]{2}{*}{$\begin{array}{c}\text { Shear stress } \\
\quad(\mathrm{Pa})\end{array}$} & \multicolumn{9}{|c|}{ Values of erythrocyte elongations índex in all blood samples aliquots } \\
\hline & Control & Fib & MDL & Timolol & Fib+MDL & $\mathbf{F i b}+\mathbf{L y}$ & $\begin{array}{c}\text { Fib+ } \\
\text { Timolol }\end{array}$ & $\begin{array}{c}\text { Fib+ } \\
\text { MDL+ } \\
\text { Timolol }\end{array}$ & $\begin{array}{c}\text { Fib+ } \\
\text { Ly+ } \\
\text { Timolol }\end{array}$ \\
\hline 0.3 & $1.76 \pm 1.29$ & $2.28 \pm 1.14$ & $2.05 \pm 1.32$ & $1.98 \pm 1.00$ & $2.38 \pm 1.13$ & $2.96 \pm 1.40$ & $2.50 \pm 1.23$ & $2.72 \pm 1.50$ & $2.75 \pm 1.72$ \\
\hline 0.6 & $4.79 \pm 1.75$ & $5.48 \pm 1.87$ & $5.71 \pm 1.64$ & $4.94 \pm 1.67$ & $6.11 \pm 1.32$ & $6.48 \pm 1.82$ & $5.72 \pm 1.94$ & $5.84 \pm 1.52$ & $6.18 \pm 2.18$ \\
\hline 1.2 & $14.1 \pm 2.62$ & $14.6 \pm 3.09$ & $15.0 \pm 2.73$ & $14.3 \pm 2.54$ & $15.2 \pm 1.83$ & $15.1 \pm 1.84$ & $14.7 \pm 2.43$ & $16.1 \pm 2.73$ & $15.0 \pm 2.39$ \\
\hline 3 & $28.9 \pm 2.78$ & $29.1 \pm 3.14$ & $29.3 \pm 2.57$ & $28.7 \pm 3.08$ & $30.0 \pm 1.97$ & $29.6 \pm 1.70$ & $29.0 \pm 2.52$ & $30.2 \pm 2.44$ & $29.2 \pm 2.25$ \\
\hline 6 & $38.6 \pm 2.24$ & $37.4 \pm 2.76$ & $38.7 \pm 2.08$ & $37.9 \pm 2.27$ & $39.2 \pm 1.81$ & $38.2 \pm 2.10$ & $38.6 \pm 2.27$ & $29.3 \pm 2.28$ & $38.6 \pm 2.51$ \\
\hline 12 & $44.7 \pm 2.62$ & $44.9 \pm 2.06$ & $44.8 \pm 2.00$ & $44.7 \pm 2.04$ & $45.6 \pm 2.29$ & $44.8 \pm 3.79$ & $45.3 \pm 2.44$ & $45.7 \pm 2.31$ & $45.2 \pm 3.69$ \\
\hline 30 & $47.7 \pm 2.94$ & $48.2 \pm 2.96$ & $47.7 \pm 3.06$ & $48.2 \pm 2.73$ & $48.7 \pm 2.75$ & $48.3 \pm 5.50$ & $48.9 \pm 3.63$ & $48.6 \pm 2.98$ & $48.7 \pm 5.17$ \\
\hline 60 & $47.7 \pm 3.11$ & $49.0 \pm 3.84$ & $48.0 \pm 3.40$ & 48.53 .00 & $48.8 \pm 3.17$ & $48.5 \pm 6.03$ & $49.2 \pm 3.99 *$ & $48.9 \pm 3.46^{*}$ & $48.9 \pm 5.8$ \\
\hline
\end{tabular}

$* \mathrm{p}<0.03$ in relation to control aliquot

Table 2. Values (Mean \pm SD) of erythrocyte nitric oxide efflux $(n M)$, GSNO $(\mu \mathrm{M})$, nitrite $(\mu \mathrm{M})$ and Nitrate $(\mu \mathrm{M})$ obtained in absence (Control) and presence of fibrinogen (Fib) or Fib plus timolol or Fib plus adenylyl cyclase inhibitor (MDL),or plus guanylyl cyclase inhibitor (Ly).

\begin{tabular}{|c|c|c|c|}
\hline Blood samples & Nitric oxide & GSNO & Nitrate \\
\hline Control & $1.09 \pm 0.11$ & $8.05 \pm 0.62$ & $9.30 \pm 0.82$ \\
\hline Fib & $1.23 \pm 0.11^{*}$ & $9.13 \pm 0.34^{*}$ & $10.15 \pm 0.64^{*}$ \\
\hline MDL & $1,17 \pm 0.22$ & $9.12 \pm 1.18^{*}$ & $10.15 \pm 0.76^{*}$ \\
\hline Timolol & $1.20 \pm 0.17$ & $10.09 \pm 0.85^{*}$ & $10.95 \pm 0.63^{*}$ \\
\hline Fib+MDL & $1.50 \pm 0.42^{*}$ & $10.86 \pm 1.29^{*}$ & $1.45 \pm 0.34$ \\
\hline Fib+Ly & $1.23 \pm 0.18$ & $11.16 \pm 1.43^{*}$ & $1.50 \pm 0.84^{*}$ \\
\hline Fib+Timolol & $1.23 \pm 0.14^{*}$ & $11.84 \pm 0.57^{*}$ & $11.55 \pm 0.69^{*}$ \\
\hline Fib+MDL+Timolol & $1.49 \pm 0.36^{*} \varphi$ & $12.28 \pm 0.78 \pm 0.67^{*}$ & $12.00 \pm 0.39^{*}$ \\
\hline Fib+Ly+Timolol & $1.28 \pm 0.15^{*}$ & $11.36 \pm 1.23^{*} \varphi$ & $10.93 \pm 0.87^{*}$ \\
\hline
\end{tabular}

In relation to the control group * $\mathrm{p}<0.02$; in relation to the Fib groups $\varphi \mathrm{p}<0.001$ and $\# \mathrm{p}<0.03$; in relation to the Fib + MDL group $\Delta \mathrm{p}<0.05$ and in relation to Fib $+\mathrm{Ly}$ group $\diamond \mathrm{p}<0.04$. 
MDL, at high shear stress, Table 1. So, cAMP synthesis, are affected by the presence of AChE- timolol less active form and by Fib-CD47 receptor complex because both type of complexes are associated to $\mathrm{Gi}$ protein and consequently adenylyl cyclase inhibition $[4,9,10]$. Our results evidenced the effect of Fib in ED is synergic with timolol plus or not with MDL because maintaining cAMP synthesis inhibited. In erythrocytes MDL activate PKC which phosphorylated PDE-3 left it in inactive state without cAMP hydrolysis [25]. The activation of PKC increased ED as showed [26]. PKC phosphorylates PTK and protein tyrosine phosphatase (PTP) proteins promoting activation and inhibition of their enzyme activities respectively resulting in band 3 phosphorylation [27]. So, in erythrocytes MDL has double function to inhibit the adenylyl cyclase enzyme and be an indirect factor of band 3 protein phosphorylation.

We verify in this study the synergic effect of Fib and timolol in terms of cAMP synthesis inhibition to be a contributing factor for the increase RBC ability to deform at high shear stress, Table 1.

The ability of erythrocyte to modify reversibly the discoid shape, allowing in vivo, its passage through the capillary network, depends on its own erythrocyte membrane cytoskeleton, internal viscosity and the area per volume ratio [28]. The integrin associate protein CD 47 is near $\mathrm{Rh}$ complex and establish interaction with 4.2 protein which in turn is associated with and ankyrin [29]. The erythrocyte cytoskeleton protein spectrin is associated with the integral band 3 protein by the ankyrin protein [30]. It was previously described by us that when the protein band 3 phosphorylation degree was modulated with inhibitors of PTK syk and PTK p53/56Lyn and of PTP, the erythrocyte elongation index (EEI) as a measure of deformability, was not affected [31]. At variance when band 3 protein is dephosphorylated by the presence of syk inhibitor plus high Fib concentrations and at low shear stress ED values are elevated [32]. The results showed in this study, Table 1, evidenced that the assemble of Fib and timolol (binding respectively to CD47 and AChE both associated to Gi protein inhibitor of adenylyl cyclase) increased ED by the sustained lower levels of cAMP.

The NO efflux from erythrocyte determined in vitro model of hyperfibrinogenemia they did not present statistical significance differences in relation to control blood aliquots [6,8,17,32]. Regarding the effects of Fib in NO efflux from erythrocytes, in the present study, we verified increased values in relation to control aliquots Table 2 . We based our explanation in the fact that band 3 protein is a transmembrane protein which co-exist in a dynamic balanced between phosphorylated and dephosphorylate degree states [33]. We have verified that when band 3 protein is phosphorylated or dephosphorylated in others studies using equal in vitro model of hyperfibrinogenemia that the values of NO efflux increased without a constant profile [6,32]. This means that Fib plus band 3 phosphorylated could induced higher or lower NO efflux values than the band 3 dephosphorylate $[6,32]$. The heterogeneity of blood samples is real and known in dependence also of the degree of hemoglobin oxygenation. Band 3 when dephosphorylated binding oxygenated hemoglobin and glycolytic enzymes [29,34]. On the other hand Fib binding CD47 may induce changes in the band 3 protein structure interaction with other cytoskeleton membrane protein and consequently phosphorylation degree modifications as mentioned above.

The present study confirms that timolol do not changes the NO efflux values in relation to those obtained with control values [13-17]. When MDL and timolol are both present in blood aliquots the NO efflux from erythrocyte increase in relation to control aliquots [17]. The present study showed that Fib affects positively the NO efflux from erythrocytes in presence of MDL without or with timolol Table 2. These results confirm previous that the high NO efflux amount from erythrocytes under high Fib levels depends on low levels of cAMP [7]. Fib did not affect the values of NO efflux verified on presence of timolol without or with guanylyl cyclase inhibitor Ly Table 2. Besides, both MDL and Ly increase the activity of PKC with consequently increased of cAMP, in turn MDL inhibits its synthesis [25] favoring the FibCD47 complex dependence of low cAMP concentration to mediate the efflux of NO from erythrocytes Table 2.

For the first time we have showed lower GSNO efflux from erythrocytes with AChE- timolol less active form than with the AChE-ACh complex active form [15]. Consequently, the amount of GSNO efflux has influence on GSNO concentration presented inside erythrocytes as evidenced in RBCs of glaucoma patients when compared with healthy donors [16]. When timolol is added to MDL or to Ly in blood aliquots the GSNO increase in relation to control aliquots [17]. In the present study timolol increase GSNO in relation to control and the positive synergic action of Fib on the GSNO values obtained in presence of timolol plus MDL in relation to control, to Fib plus MDL and to Fib plus timolol was showed in Table 2. The increase levels of GSNO result from the NO captured by reduced glutathione (GSH) which may be available from the improvement of pentose phosphate pathway at expense of glycolysis inhibition by the pyruvate kinase (PK) phosphorylation [35]. As described above PK is phosphorylated by cAMP protein kinase (cAMPK) dependent which activation results from the activation of PKC by MDL or by Ly [25].

The positive synergic effect showed by Fib when added to timolol plus MDL or Timolol plus Ly is showed in both nitrite and nitrate concentration Table 2. It is known that the binding of Fib to CD47 in erythrocytes do not induce an oxidant state in erythrocyte, as indicate by the normal peroxynitrite values evaluated [7]. Also no variation of erythrocytes peroxynitrite concentration was obtained with timolol in absence or plus MDL or plus Ly [17]. So, it is not to exclude the possible reaction of peroxynitrite with hemoglobin generates nitrate and S-nitrosohemoglobin, which liberating NO to thiol group of GSH originates GSNO [36]. The increase in nitrite and nitrate concentration obtained in the present study can be derived from the peroxynitrite decomposition [37]. These two hypothesis need to be confirmed in further study.

\section{Conclusions}

Fibrinogen increased erythrocyte deformability under timolol without or with adenylyl cyclase inhibitor MDL, at high shear stress in dependence of lower cAMP levels.

Fibrinogen plus timolol do not interfere in the effect of NO efflux from erythrocytes neither in the values of GSNO, nitrite and nitrates but in opposite those values are increase under the influence of adenylyl cyclase inhibitor, MDL.

Like for all biological parameters, it is necessary to highlight, in a simplistic way, that for each molecule component of the NO metabolism independent of the internal or external addition molecules, there are a range of initial values or control ones in dependence of the biological sample variability. From this derive the importance of our "in vitro" experimental model.

\section{Acknowledgments}

This work was funded by Fundação para a Ciência e Tecnologia: LISBOA-01-0145-FEDER-007391, project cofunded by FEDER, 
through POR Lisboa 2020 - Programa Operacional Regional de Lisboa, PORTUGAL 2020.

\section{References}

1. Mosesson MW (2005) Fibrinogen and fibrin structure and functions. $J$ Thromb Haemost 3: 1894-1904. [crossref]

2. Schechner V, Shapira I, Berliner S, Comaneshter D, Hershcovici T, et al (2003). Significant dominance of fibrinogen over immunoglobulins, C-reactive protein, cholesterol and triglycerides in maintaining increased red blood cell adhesiveness/ aggregation in the peripheral venous blood: a model in hypercholesterolemia patients. European J Cli Inv 33: 955-961.

3. Adams RA, Schachtrup C, Davalos D, Tsigelny I, Akassoglou K (2007) Fibrinogen signal transduction as a mediator and therapeutic target in inflammation: lessons from multiple sclerosis, Curr Med Chem 14: 2925-2936.

4. Oliveira S, Vitorino AV, Calado A, Rosário HS, Saldanha C (2012) Integrin-associated protein (CD47) is a putative mediator for soluble fibrinogen interaction with human red blood cells membrane. Biochim Biophys Acta 1818: 481-490. [Crossref]

5. de Oliveira S, Saldanha C (2010) An overview about erythrocyte membrane. Clin Hemorheol Microcirc 44; 63-745. [Crossref]

6. Saldanha C, Freitas T, Lopes JP, Silva AS (2014) Signal transduction pathways in erythrocyte nitric oxide metabolism under high fibrinogen levels. Korea-Australia Rheology J 26; 217-223.

7. Silva AS, Freitas T, Almeida JP, Saldanha C (2016) Fibrinogen Signaling in Erythrocyte Nitric oxide Mobilization in Presence of PI3-K and Adenylyl Ciclase Inhibitors. Eur J of Biom and Pharm Science 3; 28-34.

8. Saldanha C, Freitas T, Almeida JP (2012) Fibrinogen effects on erythrocyte nitric oxide mobilization in presence of acetylcholine. Life Sci 91: 1017-1022. [crossref]

9. Carvalho FA, Almeida JP, Fernandes I, Freitas-Santos T, Saldanha C (2009) Modulation of erythrocyte acetylcholinesterase activity and its Association with $\mathrm{G}$ protein-band 3 interactions. J Memb Biol 228: 89-97. [Crossref]

10. Carvalho FA, Almeida JP, Fernandes IO, Freitas-Santos T, Saldanha C (2008) Nonneuronal cholinergic system and signal transduction pathways mediated by band 3 in red blood cells. Clin Hemorheol Microcirc 40: 207-227. [Crossref]

11. Mesquita R, Pires I, Saldanha C, Martins-Silva J (2001) Effects of acetylcholine and spermineNONOate on erythrocyte hemorheologic and oxygen carrying properties. Clin. Hemorheol. Microcirc 25: 153-163. [Crossref]

12. Lopes de Almeida JP, Freitas-Santos, T, Saldanha C (2012) Erythrocyte deformability dependence on band 3 protein in an in-vitro model of hyperfibrinogenemia Clin Hemorh Microc 50: 213-219. [Crossref]

13. Zabala L, Saldanha C, Martins SJ, Souza RP (1999) Red blood cell membrane integrity in primary open angle glaucoma: ex vivo and in vitro studies. Eye 3: 101-103. [Crossref]

14. Saldanha C, Teixeira P, Santos FT, Napoleão P (2013) Timolol modulates erythrocyte nitric oxide bioavailability. J Clin Exp Opthalmol 4: 285.

15. Teixeira P, Napoleão $P$, Saldanha $C$ (2015) S-Nitrosoglutathione efflux in the erythrocyte. Clinic Hemorh Microc 60: 397-404. [Crossref]

16. Esteves R, Freitas T, Teixeira P, Napoleão P, Neves C, et al. (2016) Erythrocyte nitric oxide in glaucoma patients- ex vivo study Clin Hemorh Microc 64; 989-994. [Crossref]

17. Saldanha C. Santos FT, Silva AS (2018) Timolol effects on erythrocyte deformability and nitric oxide metabolism Clin Hemorh Microc 3: 144.

18. Sargento L, Saldanha C, Monteiro J, Perdigão C, Silva JM (2005) Long-term prognostic value of protein $\mathrm{C}$ activity, erythrocyte aggregation and membrane fluidity in transmural myocardial infarction Thromb Haemost 94: 380-388. [Crossref]
19. Carvalho FA, Martins SJ, Saldanha C (2004) Amperometric measurements of nitric oxide in erythrocytes Biosens. Bioelectron 20: 505-508. [Crossref]

20. Guevara I, Iwanejko J, Dembinska KA, Pankiewicz J, Wanat A, et al. (1998) Determination of nitrite/nitrate in human biological material by the simple Griess reaction Clin Chim Acta 274: 177-188. [Crossref]

21. Carvalho FA, Mesquita R, Martins SJ, Saldanha C (2004) Acetylcholine and choline effects on erythrocyte nitrite and nitrate levels. J Appl Toxicol 24: 419-427. [Crossref]

22. Cook JA, Kim SY, Teague D, Krishna MC, Pacelli R, et al. (1996) Convenien colorimetric and fluorometric assays for S-nitrosothiols, Anal Biochem 238: 150-158. [Crossref]

23. Saldanha C, Lopes JP, Freitas T, de Oliveira S, Silva AS (2010) Erythrocyte deformability responses to shear stress under external and internal Influences. Series on Biomechanics 25: 54-60.

24. Editor (2016) Ethical guidelines for publication in Clinical Hemorheology and Microcirculation Clin Hemorh Microc 63: 1-2.

25. Almeida JP, Carvalho FA, Martins SJ, Saldanha C (2008) The modulation of cyclic nucleotide levels and PKC activity by acetylcholinesterase effectors in human erythrocytes Actas Bioquimica 9: 111-114.

26. de Oliveira S, Silva AS, Saldanha C (2008) Modulation of erythrocyte deformability by PKC activity. Clin Hemorheol Microc 39: 363-373. [Crossref]

27. Zipser Y, Piade A, Barbul A, Korenstein R, Kosower NS (2002) Ca2+ promotes erythrocyte band 3 tyrosine phosphorylation via dissociation of phosphotyrosine phosphatase from band 3. Biochem J 368: 137-144. [Crossref]

28. Mohandas N, Clark MR, Jacobs MS, Shobet SB (1979) Quantitative analysis of factors regulating deformability. Blood 54: 30a.

29. Bruce LJ, Beckmann R, Ribeiro ML, Peters LL, Chasis JA, et al. (2003) A band 3-based macrocomplex of integral and peripheral proteins in the RBC membrane Blood 101: 4180-4188. [Crossref]

30. Xiu-Li A, Takakuwa Y, Nunomura W, Manno S, Mohandas N (1996) Modulation of Band 3-Ankyrin Interaction by Protein 4.1: Functional implications in regulation of erythrocyte membrane mechanical properties J Biol Chem 271: 33187-33191. [Crossref]

31. Saldanha C, Silva AS, Gonçalves S, Silva JM (2007) Modulation of erythrocyte hemorheological properties by band 3 phosphorylation and dephosphorylation. Clin. Hemorheol Microc 36: 183-194. [Crossref]

32. Lopes de A, Freitas ST, Saldanha C (2011) Evidence that the degree of band 3 phosphorylation modulates human nitric oxide efflux-in vitro model of hyperfibrinogenemia Clin Hemorh Microc 49: 407-416. [Crossref]

33. Bordin L, Ion-Popa F, Brunati AM, Clari G, Low PS (2015) Effector induced Sykmediated phosphorylation in human erythrocytes. Biochim Biophys Acta 1745: 20-28. [Crossref]

34. Bordin L, Clari G, Moro I, Vecchia FD, Moret V (1995) Functional link between phosphorylation state of membrane proteins and morphological changes of human erythrocytes Biochem Biophys 213: 249-257. [Crossref]

35. Jindal HK, Ai Z, Gascard P, Horton C, Cohen CM (1996) Specific Loss of Protein Kinase Activities in Senescent Erythrocytes. Blood 88: 1479-1487. [Crossref]

36. Gladwin MT, Wang X, Reiter CD, Yang BK, Vivas Ex (2002) S-Nitrosohemoglobin is unstable in the reductive erythrocyte environment and lacks $\mathrm{O} 2 / \mathrm{NO}$-linked allosteric function. J Biol Chem 277: 27818-27828. [Crossref]

37. Pfeiffer S, Mayer B (1998) Lack of tyrosine nitration by peroxynitrite generated at physiological pH. J Biol Chem 273: 27280-27285. [Crossref]

Copyright: (C2018 Saldanha C. This is an open-access article distributed under the terms of the Creative Commons Attribution License, which permits unrestricted use, distribution, and reproduction in any medium, provided the original author and source are credited. 